\title{
Unilateral hydrocephalus from a gangliocytoma-somatotrophinoma: first reported case
}

\author{
Simon Ryder (1) , Jed Robusto², Thomas Robertson³ , Hamish Alexander4 and Emma L Duncan ${ }^{5,6}$ \\ 1Department of Endocrinology and Diabetes, Faculty of Medicine, Royal Brisbane and Women's Hospital, University \\ of Queensland, Queensland, Australia, 2Kenneth J Jamison Neurosurgery Department, Royal Brisbane and Woman's \\ Hospital, Queensland, Australia, 3Pathology Queensland, Faculty of Medicine, Royal Brisbane and Women's Hospital, \\ University of Queensland, Queensland, Australia, 4Kenneth J Jamison Neurosurgery Department, Faculty of Medicine, \\ Royal Brisbane and Woman's Hospital, University of Queensland, Queensland, Australia, ${ }^{5}$ Department of Twin \\ Research and Genetic Epidemiology, Faculty of Life Sciences and Medicine, King's College London, London, UK, and \\ ${ }^{6}$ Faculty of Health and Medicine, Institute of Health and Biomedical Innovation, Queensland University of \\ Technology, University of Queensland, Queensland, Australia
}

Correspondence should be addressed to H Alexander Email hamish.alexander@health. qld.gov.au

\section{Summary}

Although pituitary macroadenomas often cause mass effects on surrounding structures, it is extremely rare for pituitary lesions to disturb cerebrospinal fluid circulation. Sellar gangliocytoma-pituitary adenomas (SGPAs) are also extremely rare. Here we report the unique case of a man with the unusual combination of acromegaly from an SGPA, who presented with unilateral hydrocephalus. A 60-year-old man presented with rapid neurological deterioration, bitemporal hemianopia, and acromegalic features. Neuroimaging revealed a large sellar lesion extending superiorly into the left foramen of Monro, causing acute obstructive unilateral hydrocephalus. External ventricular drain placement improved consciousness immediately. Biochemical assessment confirmed acromegaly. Following trans-sphenoidal debulking, histology revealed a mixed gangliocytoma/sparsely-granulated somatotrophinoma. Despite the residual disease, his vision recovered remarkably, low-dose cabergoline controlled residual excess growth hormone $(\mathrm{GH})$ secretion, and the residual tumour has remained extremely stable over 2 years. Hydrocephalus is an extremely rare complication of pituitary lesions, and unilateral hydrocephalus has never been reported previously. GH secretion in SGPAs is more common than for pituitary adenomas in general, raising questions regarding the aetiology and therapeutic approach to this rare combination tumour.

\section{Learning points:}

- Pituitary tumours most commonly present with symptoms related to endocrine disturbance or mass effects upon visual pathways (e.g. optic chiasm, nerves in the lateral cavernous sinus). However, extremely rarely, pituitary masses may disrupt cerebrospinal fluid (CSF) circulation resulting in hydrocephalus.

- Sellar gangliocytomas are very rare tumours and most of them are hybrid tumours with pituitary adenomas (SGPAs).

- SGPAs are typically indolent and may be functioning or non-functioning tumours.

- Growth hormone (GH)-producing SGPAs are less likely to respond to somatostatin agonists than classic somatotrophinomas.

- Primary surgical debulking via a trans-sphenoidal approach was effective in this individual, leading to the restoration of CSF circulation and improvement in visual disturbance, while also negating the need for permanent CSF diversion despite the residual tumour burden. 


\section{Background}

Pituitary adenomas are common, identified radiologically in $10-20 \%$ of individuals. The prevalence of clinically relevant pituitary adenomas is much lower, approximately $0.1 \%$ (1); fewer than half of these are macroadenomas which typically present with endocrine disturbance or mass effects such as optic chiasm compression. It is extremely unusual for macroadenomas to affect cerebrospinal fluid (CSF) circulation.

We report the extremely rare case of a man who presented with symptoms of raised intracranial pressure from unilateral obstructive hydrocephalus caused by a gangliocytoma-somatotrophinoma.

\section{Case presentation}

A 60-year-old male presented after 3 weeks of increasing confusion, headache, nausea, vomiting, ataxia, and urinary incontinence. On examination, he was drowsy, confused (Glasgow Coma Scale (GCS) 12), and obese (weight $168.1 \mathrm{~kg}$, BMI $52.5 \mathrm{~kg} / \mathrm{m}^{2}$ ), with coarse facial features, prominent supraorbital ridges, macroglossia, greasy skin, spade-like hands, and abundant axillary skin tags. CT and MRI demonstrated a large lobulated lesion arising from an expanded pituitary fossa, extending superiorly into the suprasellar cistern and left foramen of Monro, causing acute obstructive unilateral hydrocephalus (Fig. 1A and B). Emergency external ventricular drain (EVD) placement resulted in immediate improvement to GCS 14.

Further history included increasing shoe and ring sizes, severe obstructive sleep apnoea (OSA), hypertension, dyslipidaemia, erectile dysfunction, and chronic back pain. He had a poor vision with bitemporal hemianopia (Fig. 1D) but no other neurological deficits.

\section{Investigation}

Biochemical testing demonstrated elevated IGF-1 (Table 1), consistent with an integrated excess of growth hormone (GH) (i.e. acromegaly). After an oral glucose tolerance test of $75 \mathrm{mg} \mathrm{GH}$ suppressed to a nadir GH of $0.73 \mu \mathrm{g} / \mathrm{L}$, above the level suggested by some authors as a sensitive value to diagnose acromegaly ( $\mathrm{GH}$ nadir of $<0.4 \mu \mathrm{g} / \mathrm{L}$ (2)) but below the level suggested by the 2014 Endocrine Society Clinical Practice Guidelines of GH nadir of $<1 \mu \mathrm{g} / \mathrm{L}$ (3).

$\mathrm{He}$ also had mild hyperprolactinaemia and hypogonadotrophic hypogonadism, and borderline central hypothyroidism (Table 1).

\section{Treatment}

Surgery was undertaken with the primary aim of tumour debulking to re-establish CSF circulation. A soft grey tumour was removed via an endoscopic trans-sphenoidal approach, with resection limited by a descent of the diaphragma sellae.

Histologically, the tumour had two distinct cellular components (Fig. 2). The first component was monomorphous conventional pituitary adenoma cells showing weakGHexpression and numerouskeratin 'fibrous' bodies, typical of a sparsely granulated somatotrophinoma. The second component showed neuronal differentiation, with neoplastic ganglion cells including large dysmorphic forms and multinucleate forms, surrounded by a fibrillary neuropil matrix. The neuronal cells and neurofibrillary matrix were immunoreactive for NeuN and neurofilament
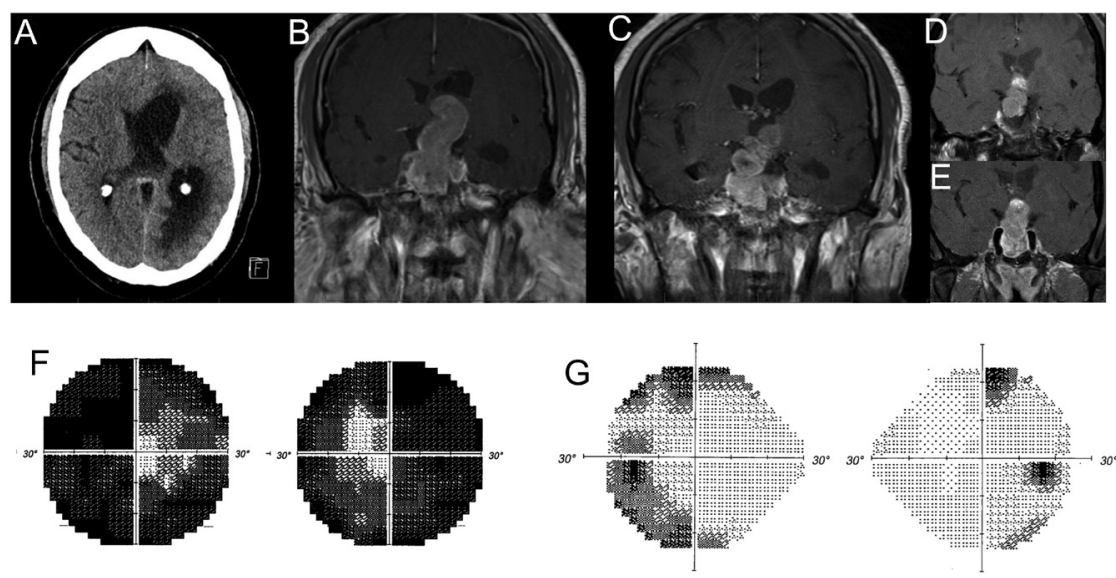

\section{Figure 1}

Cranial imaging and computerised perimetry. (A) CT scan at baseline, demonstrating unilateral hydrocephalus, trans-ependymal oedema, and a large mass arising from the pituitary fossa. (B) MRI scan after placement of EVD. A large $(23 \mathrm{~mm}$ transverse $\times 30 \mathrm{~mm}$ antero-posterior $\times 51 \mathrm{~mm}$ craniocaudal) mass arising from the pituitary fossa and extending superiorly is evident. (C) MRI scan day 3 post-operatively, demonstrating the resolution of hydrocephalus and residual tumour bulk. (D) MRI scan 12 months post-operatively, demonstrating stable residual tumour. Cabergoline commenced due to persistent IGF-1 elevation. (E) MRI scan 21 months postoperatively, demonstrating stable residual tumour. (F) Visual field assessment in the immediate postoperative period. (G) Visual field assessment 18 months postoperatively. 
Table 1 Biochemistry.

\begin{tabular}{|c|c|c|c|c|c|}
\hline Test & Reference range & $\begin{array}{c}\text { Pre-operative } \\
\text { results }\end{array}$ & $\begin{array}{c}\text { Post-operative results } \\
\text { (1-3 weeks post-op) }\end{array}$ & $\begin{array}{c}\text { Post-operative results } \\
\text { (10 months post-op) }\end{array}$ & $\begin{array}{c}\text { Post-operative results } \\
\text { (18 months) }\end{array}$ \\
\hline IGF-1 & 6.9-27 nmol/L & 64 & 39 & 33 & 23 \\
\hline $\mathrm{GH}$ & $0.05-3.00 \mu \mathrm{g} / \mathrm{L}$ & 1.09 & 0.4 & 0.13 & \\
\hline Prolactin & $56-278 \mathrm{mU} / \mathrm{L}$ & 707 & & 213 & 59 \\
\hline $\mathrm{LH}$ & $1-9 U / L$ & 0.6 & $<1$ & $<0.2$ & $<0.2$ \\
\hline $\mathrm{FSH}$ & $1-15 \mathrm{U} / \mathrm{L}$ & 1.2 & $<1$ & $<0.7$ & $<0.7$ \\
\hline Testosterone & 9-35 nmol/L & 2.0 & 0.5 & 0.5 & $0.5 *$ \\
\hline Cortisol & $140-640 \mathrm{nmol} / \mathrm{L}$ & 272 & 451 & 194 & 318 \\
\hline $\mathrm{TSH}$ & $0.3-4.5 \mathrm{mU} / \mathrm{L}$ & 0.4 & 0.5 & 0.4 & 0.7 \\
\hline Free $\mathrm{T}_{4}$ & 7.0-17 pmol/L & 8.5 & 6.7 & 9.7 & $6.7^{*}$ \\
\hline Free $T_{3}$ & 3.5-6.0 pmol/L & 3.8 & & 4.0 & \\
\hline Fasting glucose & $3.0-6.0 \mathrm{mmol} / \mathrm{L}$ & 5.3 & & 5.4 & 5.5 \\
\hline Corrected calcium & $2.10-2.60 \mathrm{mmol} / \mathrm{L}$ & 2.32 & & 2.41 & \\
\hline
\end{tabular}

*Persistent hypogonadism and hypothyroidism were due to issues with medication compliance.

FSH, follicle-stimulating hormone; GH, growth hormone; IGF-1, insulin-like growth factor 1; LH, luteinising hormone; T3, triiodothyronine; T4, thyroxine; $\mathrm{TSH}$, thyroid stimulating hormone.

protein respectively but did not express any anterior pituitary hormones (GH-releasing hormone (GHRH) was not able to be assessed). Both components expressed MAP2, but staining in the adenoma component was patchy and weaker. Ki-67 proliferative index was $<1 \%$. A final histological diagnosis was of a mixed gangliocytoma/ sparsely-granulated somatotrophinoma.

\section{Outcome and follow-up}

Postoperatively, the patient recovered well, and EVD was weaned. Post-operative MRI (day 3) demonstrated residual suprasellar tumour, as expected, but complete resolution of obstructive hydrocephalus (Fig. 1C). He was discharged within 1 week of surgery.

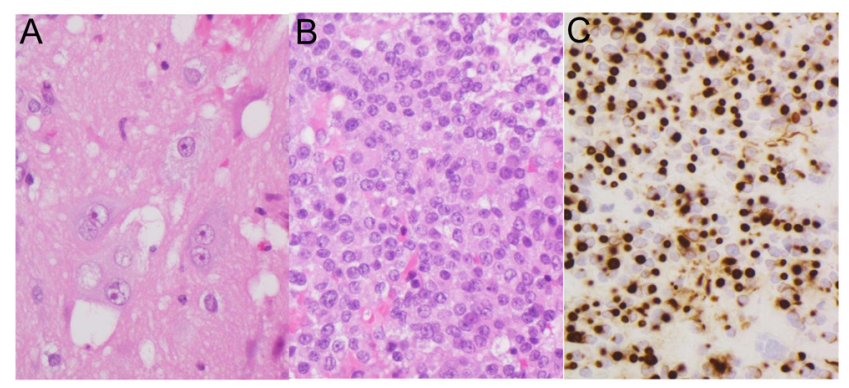

Figure 2

Histopathology. (A) Ganglion cell component comprising large, bizarre, and sometimes multinucleate neuronal cells in a fibrillary neuropil matrix (H\&E stain, 400× magnification). (B) Somatotrophinoma component with monomorphous adenoma cells (H\&E stain, 400× magnification). (C) Immunohistochemistry for keratin (CAM5.2 antibody) showing the numerous rounded 'fibrous bodies' typical of a sparsely granulated subtype of somatotrophinoma (400× magnification).
Prophylactic i.v. hydrocortisone was administered at surgical induction. Serial post-operative morning cortisol levels were robust and have remained within normal limits during follow-up. There was no pre- or postoperative evidence of posterior pituitary dysfunction (i.e. diabetes insipidus). Borderline central hypothyroidism persisted, and levothyroxine was commenced at $75 \mu \mathrm{g}$ daily. Hypogonadotropic hypogonadism also persisted, despite normalisation of prolactin levels. Testosterone was commenced around 12 months later after appropriate optimisation of OSA management (testosterone gel 1\%, initiated at half dose and up titrated gradually).

IGF-1 fell rapidly post-operatively but remained mildly elevated over many months, but rapidly normalised after commencement of cabergoline $(0.5 \mathrm{mg}$ twice weekly) (Table 1).

Computerised perimetry 2 weeks post-operatively showed persistent bitemporal hemianopia but when reassessed at 18 months had improved remarkably, with residual loss predominantly limited to a right inferior quadrantanopia (Fig. 1E). No tumour progression has been evident on imaging over 21 months.

\section{Discussion}

This is the first case report of any pituitary tumour causing unilateral hydrocephalus. Rather than symmetrical suprasellar extension through the diaphragma sellae, causing a classical 'snowman' appearance, the mixed gangliocytoma/pituitary adenoma grew unilaterally into the left foramen of Monro in an 'S' shape (Fig. 1A and B), causing an acute obstruction (evidenced by the individual's rapid clinical deterioration and trans-ependymal oedema 
on imaging). Otherwise, it behaved like most pituitary tumours, with the herniation and expansion of natural anatomical pathways rather than invasion and destruction of local structures.

Obstructive hydrocephalus from pituitary tumours is exceptionally rare. There are 29 published case reports (11 prolactinomas, 7 non-functioning pituitary adenomas, 1 GH-secreting adenoma, and a further 9 pituitary adenomas lacking detail of secretory function). There is one reported case of a sellar gangliocytoma-prolactinoma causing obstructive hydrocephalus, which exhibited a limited response to dopamine agonists (4). One giant prolactinoma required urgent EVD for symptomatic hydrocephalus (5); otherwise, affected individuals typically present with slowly progressive hydrocephalus.

Similarly, sellar gangliocytomas are very rare. A recent systematic review identified 130 published cases, mostly (85\%) coupled with pituitary adenoma (SGPAs) (6). Immunohistochemistry of the pituitary component most commonly demonstrated combined GH-PRLstaining (43\%) or GH staining alone (33\%), with prolactin-alone, ACTHalone, and non-functioning pituitary adenomas much less common. Intriguingly, immunohistochemistry of the gangliocytoma component often demonstrated GHRH positivity (positive in 28 cases, negative in 17 cases, not determined in 11 cases) (6), suggesting a possible inductive, rather than coincidental, relationship of gangliocytomas with pituitary adenomas. However, such a relationship (i.e. gangliocytoma production of a stimulatory hormone (notably, GHRH or CRH) inducing either a pituitary adenoma or pituitary hyperplasia with relevant hormonal hypersecretion) may not be straightforward $(6,7)$. GHRHstaining of the gangliocytoma did not necessarily correlate with GH secretion/staining of the pituitary adenoma; similarly, CRH staining did not necessarily correspond to ACTH secretion/staining (6). Two cases reported clinical and biochemical features of acromegaly from isolated sellar gangliocytomas with positive immunostaining for GHRH but no abnormal pituitary histopathology $(8,9)$; although the first case had insufficient pituitary tissue to assess pituitary histopathology (8) and the second acknowledged only partial pituitary resection with persistent GH hypersecretion post-operatively requiring somatostatin analogue therapy (9). We considered whether the pituitary findings in our case were somatotroph hyperplasia vs somatotrophinoma. An isolated adenoma cell showed a weak expression of PRL but this was minimal, and there was no expression of other anterior pituitary hormones (ACTH, TSH, LH, or FSH) to indicate plurihormonal production by the tumour or presence of other types of normal anterior pituitary cells, as might be expected in somatotroph hyperplasia; moreover, 'fibrous' bodies are seen in neoplastic somatotrophs and not normal or hyperplastic somatotrophs (Fig. 2C).

Thus, theaetiology ofSGPAsisunresolved.Someauthors propose that the mixed cell lineages arise from a common stem cell progenitor, given the expression of neuronalassociated markers (e.g. NFP) within adenomatous cells and, conversely, adenohypophyseal markers (e.g. keratins) within ganglion cells (10). Alternatively, a recent study of ten gangliocytoma/somatotrophinomas demonstrated the expression of the acidophilic lineage-specific transcription factor Pit-1 in a subset of ganglion cells in many of these tumours despite no GH expression, suggesting to these authors 'transdifferentiation' of neuroendocrine cells into the neuronal phenotype (11). Supporting this hypothesis, the expression of neuronal-associated markers is not unique to SGPAs and has been reported in pituitary adenomas without concomitant morphological ganglion cell differentiation (12).

This man had classical acromegalic features. His poor health literacy and distant rural location may have contributed to his late and dramatic presentation. Complete surgical resection via a single approach was not possible - and a priori not attempted. Further surgery was initially envisaged but so far has been unnecessary, with no evidence of tumour growth, stable vision, and control of excess GH secretion by cabergoline.

There is limited evidence regarding non-surgical approaches for SGPAs. Their indolent growth suggests a limited role for radiotherapy, although this might be considered for ongoing hormonal excess (6). There is one report of gamma-knife radiosurgery given 3 months post-resection in a woman with a small residual nonfunctioning SGPA who showed no evidence of tumour growth 14 months later (13); the lack of hormonal hyper-secretion at baseline and the usual indolent course of SGPAs raise questions about the necessity of this approach. Somatostatin analogues (SSAs) are of uncertain benefit. The somatotrophinoma component of GH-producing SGPAs is typically sparsely granulated (11), and this tumour subtype usually responds more poorly (at least, biochemically) to SSAs than densely granulated tumours (14). Although somatostatin receptor (SSTR) immunostaining is not routinely performed, sparsely granulated somatotrophinomas are also more likely to express SSTR-5 and rarely SSTR-2. Thus targeting SSTR-5 (e.g. by pasireotide) may be more effective than agents that predominantly target SSTR-2. There is little evidence that the ganglionic component of SGPAs will respond to 
SSAs; SSTR immunostaining of ganglionic cells has not been reported previously, although scant somatostatin positivity has been detected rarely (8).

To our knowledge, although dopamine agonist (DA) therapy is widely used either as initial or add-on therapy for somatrophinomas (3), there are no previous reports describing the use of cabergoline or other DAs in GH-producing SGPAs. There is a single report of cabergoline use in a 16-year-old boy with a sellar gangliocytoma-prolactinoma in whom cabergoline was used pre-operatively for a presumed macroprolactinoma with initial decreases in tumour size and prolactin levels. However, subsequent tumour growth after 6 months despite ongoing euprolactinaemia prompted surgical debulking and led to histological diagnosis (4). In light of the mild GH elevation, government restrictions on SSA access (usually only after DA trial), and difficulties initiating and maintaining SSAs in a geographically remote individual, cabergoline was trialed, with success. Should there be significant biochemical or tumour progression on future surveillance, then SSA therapy may be pursued.

In summary, we report the unique complication of unilateral hydrocephalus in a man with a rare mixed gangliocytoma-pituitary adenoma, in whom surgical debulking re-established CSF pathways and avoided a permanent shunt. Despite residual tumour bulk, his vision recovered remarkably and his GH excess proved remarkably simple to manage medically, and his disease has remained stable over a long period of follow-up.

\section{Declaration of interest}

The authors declare that there is no conflict of interest that could be perceived as prejudicing the impartiality of the research reported.

\section{Funding}

This research did not receive any specific grant from any funding agency in the public, commercial or not-for-profit sector.

\section{Patient consent}

Informed consent was obtained from the patient for publication of the submitted article and accompanying images.

\section{Author contribution statement}

Simon Ryder and Jed Robusto contributed equally in the preparation of this submitted article as first authors. Thomas Robertson, Hamish
Alexander and Emma Duncan contributed equally in the preparation of this submitted article as senior authors.

\section{References}

1 Lim CT \& Korbonits M. Update on the clinicopathology of pituitary adenomas. Endocrine Practice 201824 473-488. (https://doi. org/10.4158/EP-2018-0034)

2 Akirov A, Masri-Iraqi H, Dotan I \& Shimon I. The biochemical diagnosis of acromegaly. Journal of Clinical Medicine 2021101147. (https://doi.org/10.3390/jcm10051147)

3 Katznelson L, Laws Jr ER, Melmed S, Molitch ME, Murad MH, Utz A, Wass JA \& Endocrine Society. Acromegaly: an Endocrine Society clinical practice guideline. Journal of Clinical Endocrinology and Metabolism 201499 3933-3951. (https://doi.org/10.1210/jc.2014-2700)

4 Yano S, Hide T, Uekawa K, Honda Y, Mikami Y \& Kuratsu JI. Mixed pituitary gangliocytoma and prolactinoma resistant to the cabergoline treatment. World Neurosurgery 201695 620.e17-620.e22. (https://doi. org/10.1016/j.wneu.2016.08.011)

5 Scarone P, Losa M, Mortini P \& Giovanelli M. Obstructive hydrocephalus and intracranial hypertension caused by a giant macroprolactinoma. Prompt response to medical treatment. Journal of Neuro-Oncology 200676 51-54. (https://doi.org/10.1007/s11060-005-2319-0)

6 Cossu G, Daniel RT \& Messerer M. Gangliocytomas of the sellar region: a challenging diagnosis. Clinical Neurology and Neurosurgery 2016149 122-135. (https://doi.org/10.1016/j.clineuro.2016.08.002)

7 Geddes JF, Jansen GH, Robinson SF, Gomori E, Holton JL, Monson JP, Besser GM \& Révész T. ‘Gangliocytomas' of the pituitary: a heterogeneous group of lesions with differing histogenesis. American Journal of Surgical Pathology 200024 607-613. (https://doi. org/10.1097/00000478-200004000-00017)

8 Asa SL, Scheithauer BW, Bilbao JM, Horvath E, Ryan N, Kovacs K, Randall RV, Laws ER, Singer W \& Linfoot JA. A case for hypothalamic acromegaly: a clinicopathological study of six patients with hypothalamic gangliocytomas producing growth hormone-releasing factor. Journal of Clinical Endocrinology and Metabolism $1984 \mathbf{5 8}$ 796-803. (https://doi.org/10.1210/jcem-58-5-796)

9 Isidro ML, Iglesias Diaz P, Matias-Guiu X \& Cordido F. Acromegaly due to a growth hormone-releasing hormone-secreting intracranial gangliocytoma. Journal of Endocrinological Investigation 200528 162-165. (https://doi.org/10.1007/BF03345360)

10 Kontogeorgos G, Mourouti G, Kyrodimou E, Liapi-Avgeri G \& Parasi E. Ganglion cell containing pituitary adenomas: signs of neuronal differentiation in adenoma cells. Acta Neuropathologica 2006112 21-28. (https://doi.org/10.1007/s00401-006-0055-y)

11 Lopes MB, Sloan E \& Polder J. Mixed gangliocytoma-pituitary adenoma: insights on the pathogenesis of a rare sellar tumor. American Journal of Surgical Pathology 201741 586-595. (https://doi.org/10.1097/ PAS.0000000000000806)

12 Nguyen MT \& Lavi E. Pituitary adenoma-neuronal choristoma is a pituitary adenoma with ganglionic differentiation. Experimental and Molecular Pathology 201599 628-631 (https://doi.org/10.1016/j. yexmp.2015.11.004)

13 Zhou P, Ma L, Cheng S, Yin S \& Jiang S. Combined gangliocytoma and non-functioning pituitary adenoma of the pituitary gland. Neurology India 201260 311-313. (https://doi.org/10.4103/0028-3886.98522)

14 Kiseljak-Vassiliades K, Carlson NE, Borges MT, KleinschmidtDeMasters BK, Lillehei KO, Kerr JM \& Wierman ME. Growth hormone tumor histological subtypes predict response to surgical and medical therapy. Endocrine 201549 231-241. (https://doi.org/10.1007/s12020014-0383-y)

Received in final form 28 April 2021

Accepted 7 June 2021 